\title{
Will definitive chemoradiotherapy be a new normal for patients with resectable locally advanced esophageal cancer?
}

\author{
Hiroya Takeuchi ${ }^{1}$
}

Received: 29 August 2020 / Published online: 4 September 2020

(c) The Japan Esophageal Society 2020

Nowadays, neoadjuvant chemotherapy or chemoradiotherapy (CRT) followed by esophagectomy is the standard of care for patients with resectable locally advanced esophageal cancer worldwide [1-3]. On the other hand, for patients unwilling to undergo esophagectomy, definitive CRT has been an alternative treatment for those with advanced esophageal cancer [4]. However, there are several problems to be resolved for conventional definitive CRT, such as a high incidence of late toxicities and poor survival.

Japan Clinical Oncology Group (JCOG) recently conducted a multicenter prospective trial (JCOG0909) of definitive CRT followed by salvage treatment for cStage II/III esophageal squamous cell carcinoma. In the trial, the CRT regimen and salvage treatments were modified to reduce acute and late toxicities after treatments, and a new CRT protocol was chosen based on the RTOG 9405 study, which is the standard regimen in Western countries [5].

The current article by Hironaka et al. [6] reported on the comparison of the acute adverse events and efficacy of the definitive CRT among three primary tumor sites as an exploratory analysis in JCOG0909 study. Ninety-four patients $(16,59$, and 19 patients in the upper, middle, and lower thoracic groups, respectively) in total were included in the analysis. As a result, attractively, severe hematological toxicity such as Grade 3-4 leukopenia, neutropenia, and thrombocytopenia was more frequently observed in the middle and lower thoracic esophageal cancer than in the upper thoracic esophageal cancer. However, no survival difference by primary tumor sites was observed in terms of efficacy endpoints. These results might be due to the difference of radiation fields in each primary tumor site, and the study has provided a valuable signpost for clinical application of

Hiroya Takeuchi

takeuchi@hama-med.ac.jp

1 Department of Surgery, Hamamatsu University School of Medicine, 1-20-1 Handayama, Higashi-ku, Hamamatsu-shi, Shizuoka 431-3192, Japan definitive CRT in cStage II/III esophageal squamous cell carcinoma.

The 3-year overall survival curves observed in the study seemed to be acceptable when we compare with neoadjuvant chemotherapy or CRT followed by esophagectomy, suggesting that modified definitive CRT with optimal salvage treatment would be a promising option for patients with cStage II/III esophageal squamous cell carcinoma who do not want to undergo upfront esophagectomy. Further studies such as exploration of molecular markers which can predict the response to definitive CRT accurately is needed, but the definitive CRT with salvage treatment may become a new standard as esophagus-preservation therapy in resectable locally advanced esophageal cancer.

Esophagus, the official journal of the Japan Esophageal Society, provides significant studies as one of the leading journals in the fields of benign and malignant diseases of the esophagus. The impact factor reached 3.130 in 2019. The journal welcomes original articles, review articles, and short articles including technical notes and letters to the editor, which will contribute to a "new normal" in esophageal studies.

\section{Compliance with ethical standards}

Ethical statement This article does not contain any studies with human or animal subjects performed by the author.

Conflict of interest I have no conflict of interest for my manuscript.

\section{References}

1. Sjoquist KM, Burmeister BH, Smithers BM, et al. Survival after neoadjuvant chemotherapy or chemoradiotherapy for resectable oesophageal carcinoma: an updated meta-analysis. Lancet Oncol. 2011;12:681-92. 
2. Ando N, Kato H, Igaki $\mathrm{H}$, et al. A randomized trial comparing postoperative adjuvant chemotherapy with cisplatin and 5-fluorouracil versus preoperative chemotherapy for localized advanced squamous cell carcinoma of the thoracic esophagus (JCOG9907). Ann Surg Oncol. 2012;19:68-74.

3. Van Hagen P, Hulchof MC, van Lanschot JJ, et al. Preoperative chemoradiotherapy for esophageal or junctional cancer. N Engl J Med. 2012;366:2074-84.

4. Hironaka S, Ohtsu A, Boku N, et al. Nonrandomized comparison between definitive chemoradiotherapy and radical surgery in patients with $\mathrm{T}(2-3) \mathrm{N}($ any) $\mathrm{M}(0)$ squamous cell carcinoma of the esophagus. Int J Radiat Oncol Biol Phys. 2003;57:425-33.

5. Minsky BD, Pajak TF, Ginsberg RJ, et al. INT 0123 (Radiation Therapy Oncology Group 94-05) Phase III trial of combined modality therapy for esophageal cancer: high-dose versus standard-dose radiation therapy. J Clin Oncol. 2002;20:1167-74.

6. Hironaka S, Komori A, Machida R, et al. The association of primary tumor site with acute adverse event and efficacy of definitive chemoradiotherapy for cStage II/III esophageal cancer: an exploratory analysis of JCOG0909. Esophagus. 2020. https://doi. org/10.1007/s10388-020-00741-w.

Publisher's Note Springer Nature remains neutral with regard to jurisdictional claims in published maps and institutional affiliations. 